\title{
Implementación de la Realidad Aumentada en un sistema opto mecatrónico de grabado láser
}

\section{Augmented Reality implementaction into an opto mechatronic laser engraver system}

BLANCO-MIRANDA, Alan David †*, GARCÍA-CERVANTES, Heraclio y ANDRADE-ARENAS, Iván

Universidad Tecnológica de León

ID $1^{\text {er }}$ Autor: Alan David, Blanco-Miranda / ORC ID: 0000-0002-8595-8634, Researcher ID Thomson: W-9701-2019, CVU CONACYT ID: 298274

ID $1^{\text {er }}$ Coautor: Heraclio, García-Cervantes / ORC ID: 0000-0002-4229-9229, Researcher ID Thomson: X-5622-2019, CVU CONACYT ID: 290829

ID $2^{\text {do }}$ Coautor: Iván, Andrade-Arenas / ORC ID: 0000-0003-4004-4166, Researcher ID Thomson: X-6374-2019, CVU CONACYT ID: 1014567

DOI: $10.35429 / \mathrm{JCS} .2019 .8 .3 .24 .32$

Recibido: 12 de Marzo, 2019; Aceptado 30 de Junio, 2019

\section{Resumen}

El objetivo principal del presente trabajo es el de desarrollar una aplicación móvil interactiva para el monitoreo, descripción y capacitación en el manejo de una máquina de grabado láser haciendo uso de la tecnología de realidad aumentada. Se hace uso del diseño CAD de la máquina generando una visualización de cada uno de los elementos que la conforman con una descripción de sus principales características. También, se incluye una opción donde se puede observar la animación 3D del proceso de grabado. Todo lo anterior se genera mediante el uso de software libre (Unity, Vuforia y C\#) creando así una opción práctica para la capacitación en el manejo de este tipo de maquinaria. Este tipo de tecnología se puede implementar en diferentes entornos tanto educativos como industriales. Para la comodidad de uso, la aplicación generada cuenta con un entorno virtual amigable para el usuario y se desarrolla para las principales plataformas digitales (Android, iOS, Windows).

Realidad Aumentada, Capacitación, Sistema de grabado laser

\begin{abstract}
The main objective of this work is to develop an interactive mobile application for monitoring, description and training in the operation of a laser engraving machine using augmented reality technology. The CAD design of the machine is used, generating a visualization of each of the elements that make it up with a description of its main characteristics. Also, an option is included where you can observe the 3D animation of the engraving process. All of the above is generated through the use of open source software (Unity, Vuforia and C \#) thus creating a practical option for training in the handling of this type of machinery. This type of technology can be implemented in different educational and industrial environments. For the convenience of use, the generated application has a user-friendly virtual environment and is developed for the main digital platforms (Android, iOS, Windows).
\end{abstract}

Augmented reality, Training, Laser engraving system

Citación: BLANCO-MIRANDA, Alan David, GARCÍA-CERVANTES, Heraclio y ANDRADE-ARENAS, Iván. Implementación de la Realidad Aumentada en un sistema opto mecatrónico de grabado láser. Revista de Simulación Computacional. 2019. 3-8: 24-32

\footnotetext{
* Correspondencia al Autor: (ablanco@utleon.edu.mx)

$\dagger$ Investigador contribuyendo como primer autor.
} 


\section{Introducción}

El hombre se ha dado cuenta de la importancia de realizar mediciones. Medir es aprender, con lo cual se genera una mayor cantidad de información la cual ayuda en el proceso de entendimiento y toma de decisiones acertadas en la transformación y convivencia con la naturaleza. Es por esto que ha existido la necesidad e interés por estudiar y desarrollar instrumentos además de técnicas de medición en los distintos campos de la ciencia y la tecnología, las cuales permitan analizar, caracterizar y visualizar un amplio espectro de fenómenos físicos y procesos industriales.

Las técnicas de Ingeniería Inversa, Realidad Aumentada y Realidad Virtual [1-4] han sido ampliamente utilizadas para la obtención de la forma de un objeto y su descripción en ambientes interactivos con el usuario, provocando una sensación totalmente inmersiva y de interacción con los procesos reales.

Las características que hacen a estas técnicas tan importantes en este tipo de trabajos de desarrollo tecnológico es que utilizan un ambiente virtual y de amplia seguridad para el usuario ya que no entran en contacto físico con el ambiente analizado, y por consiguiente son no destructivas, pues no causan ninguna fractura $o$ destrucción de la zona de trabajo.

Finalmente, otra característica muy importante es que proveen una muy alta sensibilidad, con lo que se pueden realizar mediciones y capacitaciones a un gran número de personas, aunado a su factibilidad de entregar resultados de campo completo.

Debido a las facilidades y ventajas que presentan estas técnicas, es que son utilizadas para la reducción de la brecha entre los modelos educativos actuales y los ambientes industriales, permitiendo conocer información sobre características específicas, como caracterizar sus materiales, monitorear distintos procesos y sin la necesidad de contar con el equipo físicamente.

Habiendo revisado trabajos similares [], se desea continuar implementar el uso de la técnica de realidad aumentada para visualizar el proceso de grabado de una máquina láser y en este caso ahondar en el campo de capacitación en el manejo de maquinaria especializada, así como monitorear los componentes de la misma y función desempeñada.
$\mathrm{Al}$ tratarse de una máquina de desarrollo propio, se pretende realizar un análisis adecuado en la obtención de información, haciendo uso del diseño digital (por sus siglas en inglés, CAD) y con él, lograr obtener una similitud al proceso real de funcionamiento generando un sistema de capacitación amigable para el usuario.

Dentro de los resultados reportados se muestra el funcionamiento en realidad aumentada de una máquina $\mathrm{CNC}$ de grabado láser, programa de capacitación sobre uso y sus planes de mantenimiento, además del diseño 3D del modelo.

El presente trabajo se divide en 3 principales secciones, la primera de ellas describe la metodología en la cual se basa la tecnología de la realidad aumentada haciendo referencia a trabajos previos realizados en este campo de investigación. La segunda muestra los resultados obtenidos y las funciones disponibles en la aplicación generada. Finalmente la tercera sección muestra las conclusiones del trabajo y hace una extensión al trabajo futuro de este proyecto.

\section{Marco Teórico}

En la presente sección se describen las principales características tanto cualitativas como cuantitativas de la técnica de realidad aumentada. La realidad aumentada se define como la integración de información digital a una imagen del mundo real a través de la cámara de los dispositivos tecnológicos.

Un sistema de realidad aumentada toma como referencia una imagen, la cual es procesada por un algoritmo de detección de bordes [6-8], el cual prepara la imagen para la etapa de segmentación y el reconocimiento de patrones [9-10]. Este proceso permite determinar la posición en la cual se debe remplazar un objeto real por uno virtual, tomando en cuenta su posición y perspectiva [11].

Una característica que ofrece la técnica de realidad aumentada es que permite incorporar objetos reales con virtuales en un mismo entorno, brindando el uso de artefactos reales para manipulación de objetos virtuales generando así el uso de esta tecnología como una plataforma para la interacción hombre-máquina. 
Esta tecnología presenta un gran potencial, y la convierte en una las principales herramientas para la innovación tecnológica en los próximos años. Sus principales ventajas sobre otras técnicas [5] es que permite optimizar tiempos en las tareas diarias de los operadores, ya que brinda información visual y precisa de las acciones a desarrollar. Además, el usuario tiene al alcance la visualización de la descripción dada.

Las aplicaciones de realidad aumentada son vastas abarcando diferentes áreas, educación [12-14], industriales [15-17], capacitación [18] publicidad [19], médicas [20-22], entre otras, donde el usuario puede convertirse en el protagonista al interactuar con la aplicación.

\section{Metodología a desarrollar}

Las tareas típicas de entornos en la Industria 4.0 deben combinar varios aspectos, uno de ellos es contemplar que la distancia del objeto aumentado al usuario puede variar de magnitud (varios centímetros a varios metros), otro es que el objeto virtual también puede variar en tamaño, además de que puede tener varias formas y texturas. Otro parámetro a tener en cuenta son las condiciones de iluminación y sonido en el ambiente de trabajo ya que pueden afectar la visualización del sistema.

Cuando las aplicaciones de AR se usan en el ciclo de diseño centrado en el usuario (UCD, por sus siglas en ingles), necesitan apoyarse en la observación y el análisis de la interacción del usuario a través de la recopilación de datos. En la figura 1 se muestra el proceso general por etapas para generar la AR de un objeto.

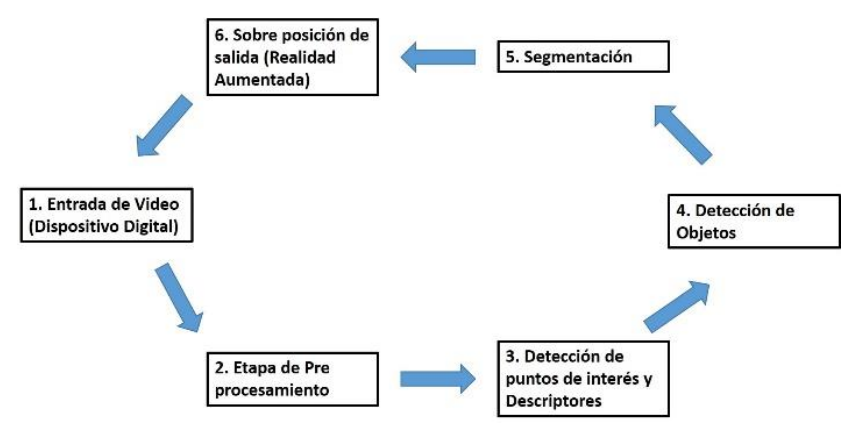

Figura 1 Proceso para generar la Realidad Aumentada de un objeto
Se debe tener en cuenta la complejidad de los datos en los procesos de fabricación reales para la creación de prototipos de alta fidelidad que permitan su elaboración y la posibilidad de desarrollo iterativo.

Dentro de estas aplicaciones AR para cualquier usuario se debe proporcionar la visualización de datos y puede permitir también la entrada del usuario (por ejemplo, caso de interacción táctil de teléfonos móviles y tabletas).

\section{Etapa de pre-procesamiento}

Cada cuadro de la secuencia de video se captura y se convierte en su equivalente en escala de grises usando filtros para la eliminación de ruido presente en la imagen.

\section{Detección de puntos de interés y uso de descriptores}

Parte de la técnica de AR es codificar una estructura local distintiva en una imagen que pueda usarse como parámetro para determinar otras características de interés.

Los detectores y descriptores de funciones son de gran utilidad en aplicaciones de reconocimiento de objetos, reconstrucción 3D, entre otros. Los puntos de interés se determinan en varias escalas y orientaciones. Un punto característico que requiere la $\mathrm{AR}$, es la postura invariable y generar resultados aceptables para la escala, orientación y posición.

También en necesario tener una alta capacidad de detección incluso cuando cambian las condiciones de visualización y reducir el número de falsos positivos. Un descriptor de características debe ser distintivo e insensible a la deformación local de la referencia. Los parámetros clave a considerar en cada modelo son la ubicación, la escala y la orientación [23].

\section{Detección de objeto}

Los pasos a seguir para la detección del objeto son los siguientes: 1. Suavizado a la imagen, 2. Proceso de correlación para subdividir la imagen en bloques cuadrados. 3. Generación de un histograma de orientación de borde a partir de cada pixel. 4. Normalizar los bloques descriptores llamados histograma de gradientes orientados (HOG, por sus siglas en inglés). 
La ventana de detección tiene una cuadrícula superpuesta de estos descriptores HOG.

En esta etapa también se pueden utilizar diferentes clasificadores, como un clasificador lineal SVM para diferenciar los objetos humanos y no humanos [24].

\section{Segmentación}

La segmentación es el proceso de asignar una etiqueta a cada píxel en una imagen de manera que los píxeles con la misma etiqueta compartan ciertas características.

El resultado de la segmentación es un conjunto de segmentos que cubren colectivamente toda la imagen, o un conjunto de contornos extraídos de la misma. Cada uno de los píxeles en una región es similar con respecto a alguna propiedad característica o calculada, como el color, la intensidad o la textura. Las regiones adyacentes son significativamente diferentes con respecto a las mismas características [25]. Cuando esta etapa se aplica a un conjunto de imágenes, como se usa en el área médica, los contornos resultantes se pueden usar para crear reconstrucciones 3D con la ayuda de algoritmos de interpolación [26].

\section{Sobre posición de salida}

El valor principal de la $\mathrm{AR}$ es la forma en que los componentes del mundo digital se mezclan con la percepción que una persona tiene del mundo real, no como una simple visualización de datos, sino a través de la integración de sensaciones inmersivas, que se perciben como partes naturales de un ambiente.

La información sobre el medio ambiente y sus objetos se superpone en el mundo real. Esta información puede ser virtual o real. La AR también tiene mucho potencial en la recopilación y el intercambio de conocimiento tácito. Las técnicas de aumento generalmente se realizan en tiempo real y en contextos semánticos con elementos del entorno.

En la figura 2 se muestra el esquema del funcionamiento de un sistema de AR en general, donde se observa la relación entre la aplicación final y el sistema ciber físico y como se relaciona con las etapas de sincronización y visualización para el usuario.

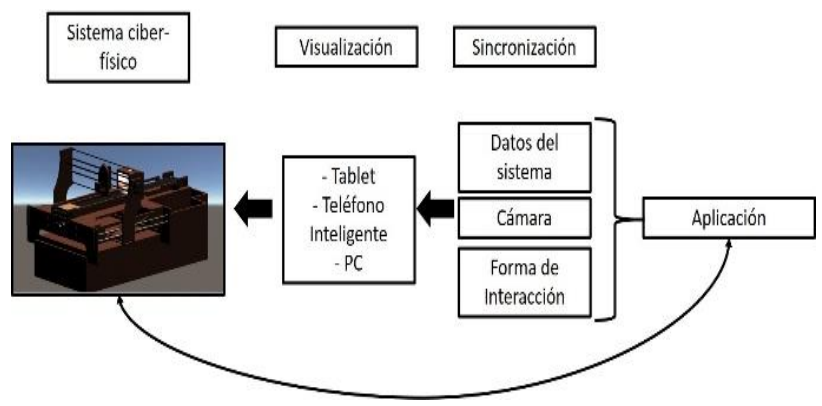

Figura 2 Esquema de funcionamiento e interacción entre usuario y sistema AR

\section{Resultados}

En esta sección se presentan los principales resultados obtenidos, describiendo la interfaz final desarrollada y su implementación en un sistema Android, lo cual funge como un apoyo en la capacitación para el manejo del sistema de grabado láser. El modelo CAD se adaptó al motor de desarrollo, Unity, haciendo uso de la extensión de archivo obj, cumpliendo con las condiciones de compatibilidad entre software [27-28]. Además, esta extensión permite la exportación de manera conjunta de las texturas y materiales del modelo 3D original.

La transferencia se llevó a cabo exportando cada una de las piezas del modelo de manera individual, generando así un mejor control y gestión del sistema original dentro de Unity. En la Figura 3, se muestra el modelo original creado en el software CAD (Autodesk Inventor), sin sufrir alguna modificación.

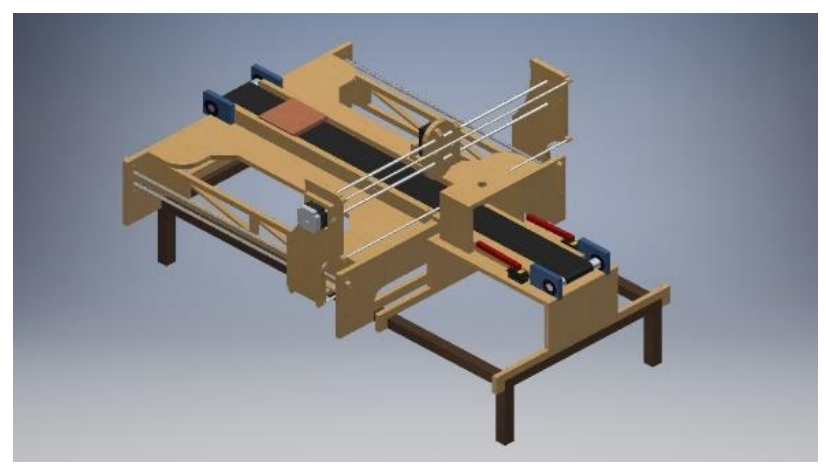

Figura 3 Modelo original diseñado en software CAD (Autodesk Inventor - Versión Estudiantil)

Sin embargo, la estructura física final tuvo modificaciones en su configuración para optimizar su funcionamiento y mediante el uso de la interfaz gráfica del motor de desarrollo Unity, Figura 4, se pueden observar las partes agregadas al modelo 3D. 


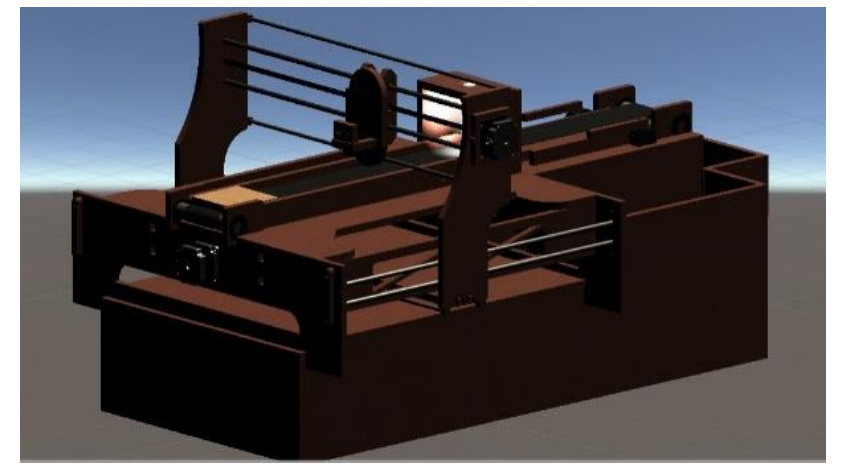

Figura 4 Modelo 3D modificado en Unity con adecuaciones a sistema físico

Los paneles laterales que cubren los soportes de la estructura de la máquina, además los cajones que se encuentran al final de la trayectoria de la banda transportadora fueron colocados. Así mismo se modificó la ubicación del cuarto o el soporte de la cámara.

Con la finalidad de replicar la apariencia de la máquina, como se puede observar en la figura $5 \mathrm{a}$. La figura $5 \mathrm{~b}$ muestra la interfaz de control automático para la clasificación de las piezas por color, forma y tamaño.

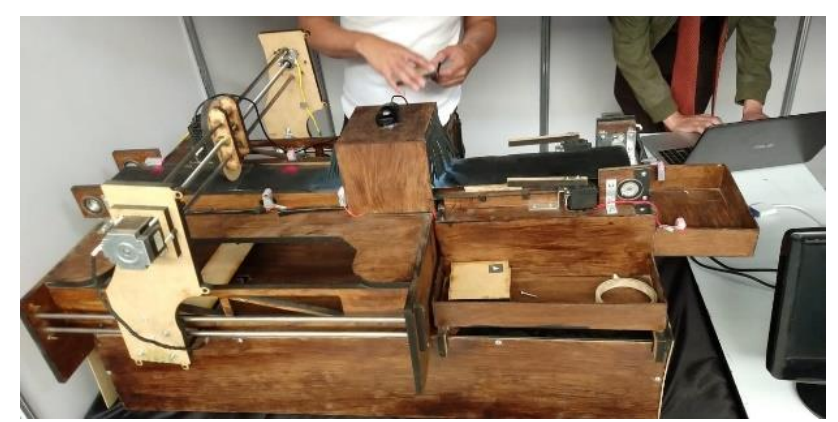

a)

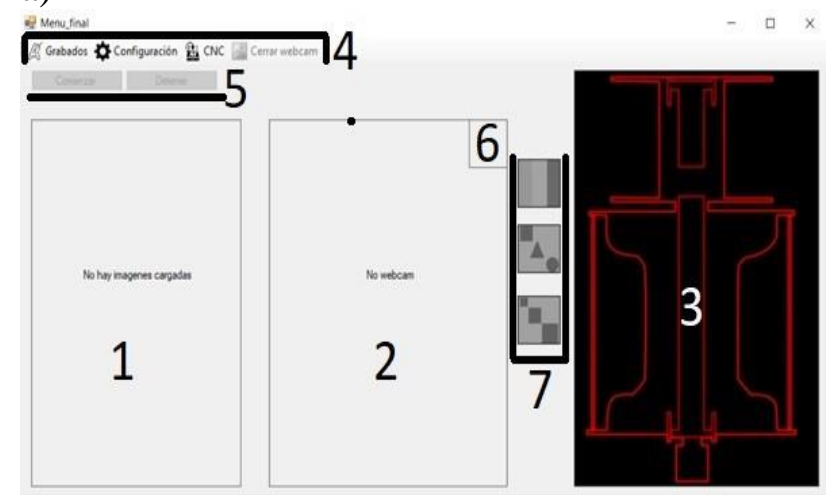

b)

Figura 5 a) Modelo físico del sistema opto mecatrónico de grabado láser; b) Interfaz de control de grabado (1 Imagen a grabar, 2 - Imagen procesada en tiempo real, 3Estado del sistema, 4 -Barra de Menús, 5 - Botones de activación de sistema, 6 - Icono del tipo de clasificación seleccionada, 7- tipo de clasificación deseada)
Además, se realizó la simulación del sistema láser y la iluminación del sistema de visión mediante el uso de los componentes Line Renderer y Spot Light [29-30] generando un entorno virtual similar al sistema real. (Figura $6 \mathrm{a}$ y $6 b)$

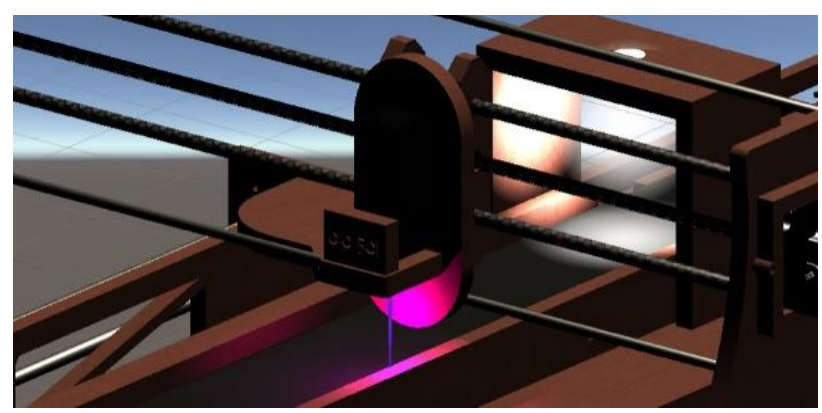

a)

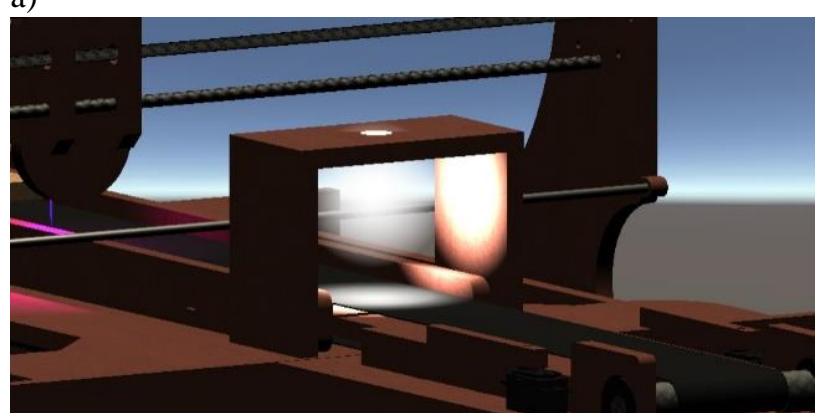

b)

Figura 6 a) Simulación de sistema láser; b) Simulación de iluminación en sistema de visión automatizado

En la figura 7, se muestra la animación del despiece de las zonas más destacadas de la máquina de grabado láser.

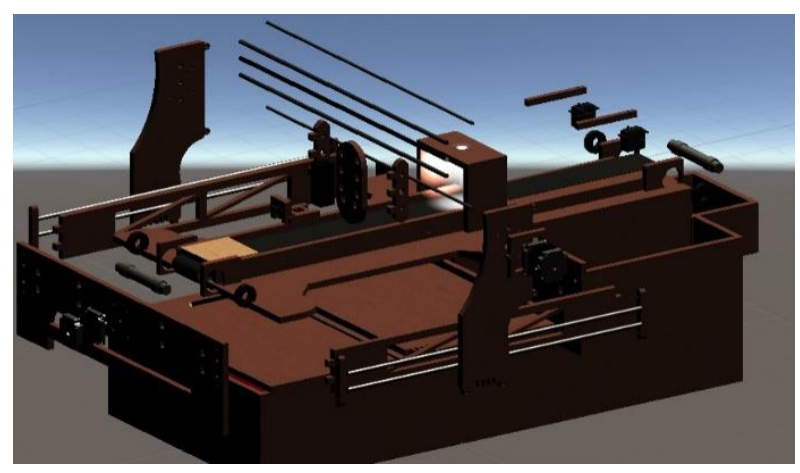

Figura 7 Modelo 3D del despiece del sistema de grabado láser

En la figura $8 \mathrm{a}-\mathrm{g}$ se muestran las secuencias de la animación que simula el funcionamiento del proceso de grabado láser del sistema opto mecatrónico. 

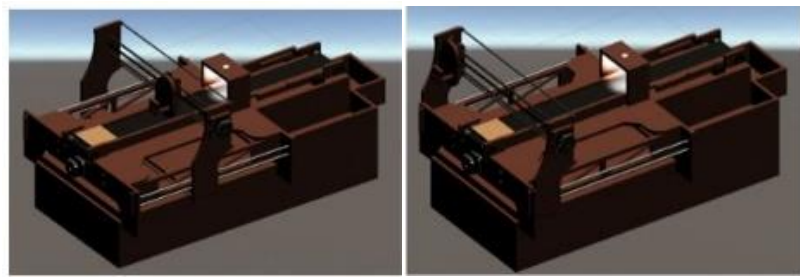

a )

b )

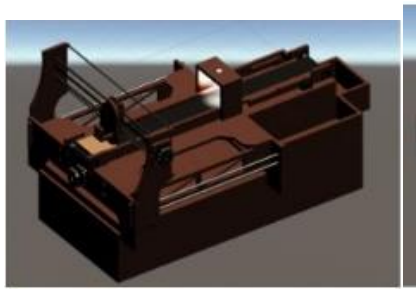

c)

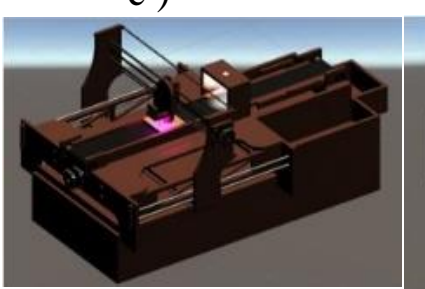

e )

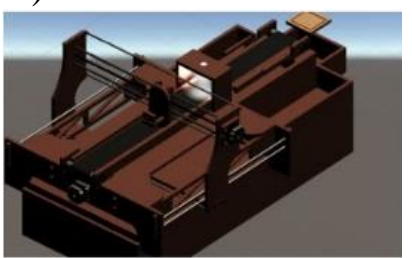

g )

Figura 8 a-g Secuencia de animación del proceso de grabado láser en una pieza de madera y su clasificación en base a forma

Las herramientas y funcionalidades diseñadas para la aplicación, fueron creadas mediante los elementos de Interfaz de Usuario (UI, por sus siglas en inglés) de Unity y programadas en el lenguaje C\#. En la tabla 1, se muestran las herramientas y la función de cada una de estas dentro de la aplicación.

\begin{tabular}{|c|c|c|}
\hline no & $\begin{array}{l}\text { Nombre de la } \\
\text { herramienta }\end{array}$ & Descripción \\
\hline & Salir & $\begin{array}{l}\text { Permite al usuario cerrar } \\
\text { la aplicación. }\end{array}$ \\
\hline & Flash & $\begin{array}{lrr}\text { Enciende el } & \text { flash } & \text { del } \\
\text { dispositivo, útil } & \text { para } \\
\text { condiciones } & \text { de } & \text { poa } \\
\text { iluminación } & \text { en } & \text { el } \\
\text { ambiente } & & \\
\end{array}$ \\
\hline & Opciones & $\begin{array}{lrr}\text { Despliega el menú } & \text { me } \\
\text { secundario, donde } & \text { se } \\
\text { muestran } & & \text { las } \\
\text { funcionalidades de la } \\
\text { aplicación. }\end{array}$ \\
\hline
\end{tabular}

Tabla 1 Herramientas y descripción de la pantalla principal de la aplicación móvil
En la figura 9, se observa la interfaz primaria de la aplicación que está compuesta por una barra principal donde se ubican las herramientas principales y una barra secundaria donde el logo de la UTL está ligado a la página web oficial de la universidad.

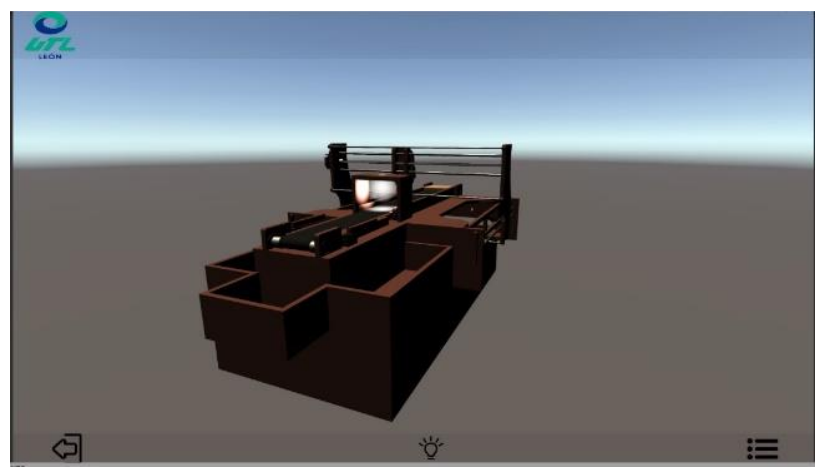

Figura 9 Interfaz primaria del sistema de grabado láser, al centro el modelo de la máquina, en la parte inferior la barra de menús descrita en la tabla 3 y en la parte superior el logo de la UTL

El contenido del menú secundario presenta los botones virtuales, los cuales activan la secuencia de la animación, así como el despliegue de las ventanas de mantenimiento e ítems, como se muestra en la figura 10.

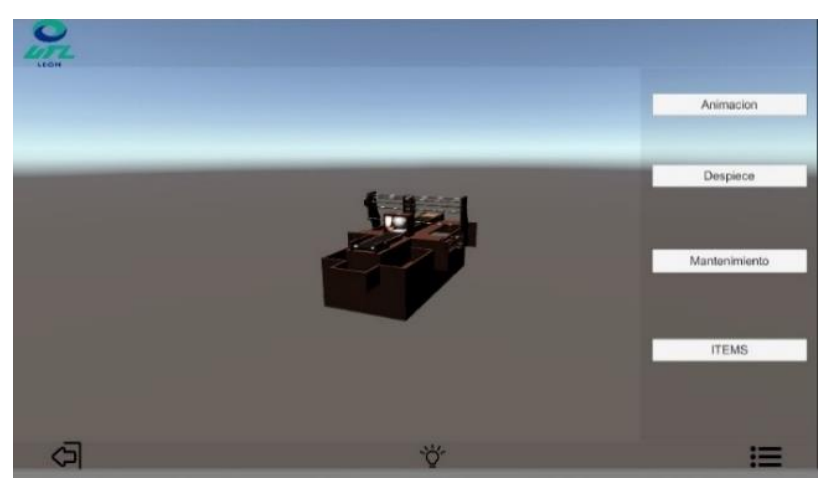

Figura 10 Interfaz secundaria de sistema de realidad aumentada. A la derecha los botones para iniciar la animación del sistema, separación en piezas, planes de mantenimiento (preventivo, predictivo y correctivo) de los principales elementos e ítems de las piezas con sus principales características

El target de la aplicación se diseñó buscando representar la ideología y el entorno que representa el aula STEM de la universidad. La figura 11, muestra el resultado del diseño final. Este target se emplea como objetivo para el despliegue de la realidad aumentada al estar enfocado en la cámara del dispositivo digital. 


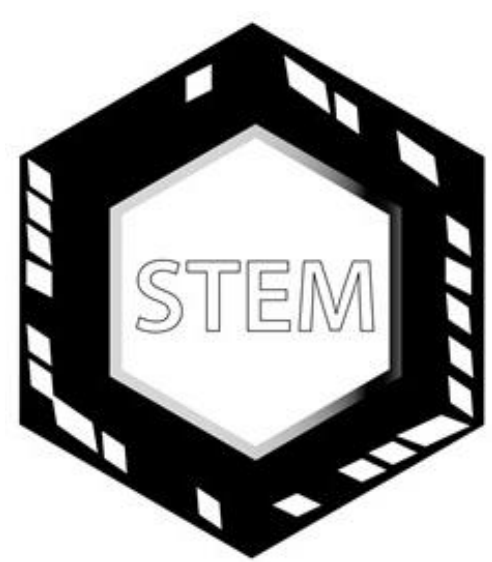

Figura 11 Diseño del target de referencia para la cámara del dispositivo donde se instale la aplicación

Con la finalidad de mostrar la funcionalidad de la aplicación generada, se desarrolló un video donde se muestra el funcionamiento de la aplicación móvil. Si se desea visualizar dicho video se deja el siguiente código QR.

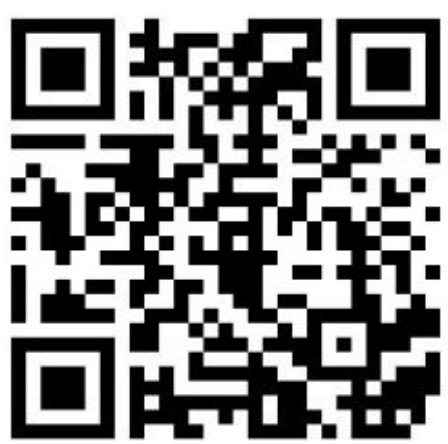

Figura 12 Código QR de video demostrativo de aplicación en funcionamiento

Por lo tanto, se logró obtener el desarrollo del entorno virtual y de la aplicación de realidad aumentada en conjunto con las características principales del sistema opto mecatrónico de grabado láser por $\mathrm{CNC}$.

Como resultado final se generó un archivo ejecutable para dispositivos digitales Android el cual puede ser implementado en cualquier lugar, siempre y cuando se tenga el target correspondiente.

Debido a razones de distribución y nuevas políticas en el uso de licencias de Vuforia [31], la aplicación deberá ser instalada directamente en el dispositivo por medio de conexión USB y no desde la App Store de Google como las aplicaciones comerciales.
Cabe señalar que esta aplicación de realidad aumentada se encuentra abierta a cualquier persona que desee visualizar y conocer las principales características de un sistema opto mecatrónico de grabado laser tipo CNC.

\section{Conclusiones}

Con ayuda del diseño CAD como fuente de proyección para la realidad aumentada y software libre de desarrollo personal (Unity), se crearon diferentes conexiones entre el diseño y sus características añadidas, esto con la finalidad de darle una visión más cercana al modelo real y lograr así una mejor experiencia para el usuario. Además, se implementaron las diferentes funciones con la finalidad de generar los elementos de una aplicación de calidad mediante la cual se acceda al sistema de capacitación de manera sencilla.

Con el propósito de evitar discrepancias entre el mundo real y el sistema virtual, se generó una referencia gráfica, la cual permite tener su implementación en cualquier espacio disponible, incluso sin la presencia de la máquina física.

Finalmente, a la aplicación generada, como se mencionó anteriormente, se le dio la cualidad de ser ejecutada en cualquier dispositivo inteligente, ya sea celular, Tablet o PC con sistema operativo Windows y/o Android, generando así una mayor portabilidad del producto.

Por último, se generó un producto completo el cual será la base de un desarrollo de mayor alcance que contará con una segunda etapa en donde se implementará la realidad virtual con el objetivo de generar una experiencia totalmente inmersiva para el usuario haciendo uso del sistema HTC VIVE [32-33].

\section{Agradecimientos}

En la realización del presente trabajo se contó con el total apoyo de la Universidad Tecnológica de León. Se agradece a los alumnos del grupo de TSU en Optomecatrónica por la realización de la máquina CNC y la aportación al desarrollo del sistema físico. 


\section{Referencias}

[1] Azuma R., Baillot Y., Behringer R., Feiner S., Julier S., MacIntyre B. (2001). Recent advances in augmented reality. IEEE Comp. Graph. Appl. 21 34-47. 10.1109/38.963459.

[2] Carmigniani J., Furht B., Anisetti M., Ceravolo P., Damiani E., Ivkovic $M$. (2011). Augmented reality technologies, systems and applications. Multimed. Tools Appl. 51 341-377. 10.1007/s11042-010-0660-6.

[3] Heras. L., Villareal, J. (2004). La realidad aumentada: una tecnología en espera de usuarios. Revista Digital Universitaria, 8(6). Recuperado de http://www.revista.unam.mx/vol.8/num6/art48/j un_art48.pdf.

[4] Hernandez Maldonado, V., Hernandez Salinas, V. (2016). La realidad aumentada. [INFOTEC-CONACYT] Recuperado de https://centrosconacyt.mx/objeto/realidadaumen tada/.

[5] Zyda M. (2005). From visual simulation to virtual reality to games. Computer 38 25-32. 10.1109/MC.2005.297.

[6] Pulkit Khandelwal, P. Swarnalatha, Neha Bisht, S. Prabu, Detection of Features to Track Objects and Segmentation Using GrabCut for Application in Marker-less Augmented Reality, Procedia Computer Science, Volume 58, 2015, Pages 698-705, ISSN 1877-0509, 10.1016/j.procs.2015.08.090.

[7] V. Sowmya, Neethu Mohan, K.P. Soman, Edge Detection Using Sparse Banded Filter Matrices, Procedia Computer Science, Volume 58, 2015, Pages 10-17, ISSN 18770509, 10.1016/j.procs.2015.08.003.

[8] Zhang, B., (2017). Design of mobile augmented reality game based on image recognition, EURASIP Journal on Image and Video Processing. 2017(1) 90. 10.1186/s13640017-0238-6.

[9] Li, W., Nee, A. Y. C., Ong, S. K. (2017). A State-of-the-Art Review of Augmented Reality in Engineering Analysis and Simulation, Multimodal Technologies and Interaction, 1(3). 10.3390/mti1030017.
[10] Chari V, Singh J M, Narayanan P J, (2008) Augmented Reality using OverSegmentation, National Conference on Computer Vision Pattern Recognition Image Processing and Graphics.

[11] Ganzha M., Maciaszek L., Paprzycki M. (2015). Augmented Reality Using Optical Flow, Proceedings of the 2015 Federated Conference on Computer Science and Information Systems, ACSIS 5 841-847. 10.15439/2015F202.

[12] Di Serio Á., Ibáñez M. B., Kloos C. D. (2013). Impact of an augmented reality system on students' motivation for a visual art course. Comput. Educ. 68 586-596. 10.1016/j.compedu.2012.03.002.

[13] Bacca J., Baldiris S., Fabregat R., Graf S. (2014). Augmented reality trends in education: a systematic review of research and applications. J. Educ. Technol. Soc. 17133.

[14] Brown A., Green T. (2016). Virtual reality: low-cost tools and resources for the classroom. TechTrends 60 517-519. 10.1007/s11528-016-0102-z.

[15] Chen Y. C., Chi H. L., Hung W. H., Kang S. C. (2011). Use of tangible and augmented reality models in engineering graphics courses.J. Prof. Issues Eng. Educ. Pract. 137 267-276. 10.1061/(ASCE)EI.19435541.0000078 .

[16] Choi S., Jung K., Noh S. D. (2015). Virtual reality applications in manufacturing industries: past research, present findings, and future directions. Concurr. Eng. 23 40-63. 10.1177/1063293X14568814.

[17] Feiner S., MacIntyre B., Hollerer T., Webster A. (1997). A touring machine: prototyping 3D mobile augmented reality systems for exploring the urban environment, in Digest of Papers. First International Symposium on Wearable Computers, (Cambridge, MA: IEEE; ), 74-81. 10.1109/ISWC.1997.629922.

[18] Schwald B., De Laval B. (2003). An augmented reality system for training and assistance to maintenance in the industrial context. J. WSCG 11. 
[19] Song H., Chen F., Peng Q., Zhang J., Gu P. (2017). Improvement of user experience using virtual reality in open-architecture product design. Proc. Inst. Mech. Eng. B J. Eng. Manufact. 232.

[20] Baus O., Bouchard S. (2014). Moving from virtual reality exposure-based therapy to augmented reality exposure-based therapy: a review. Front. Hum. Neurosci. 8:112. 10.3389/fnhum.2014.00112.

[21] Bohil C. J., Alicea B., Biocca F. A. (2011). Virtual reality in neuroscience research and therapy. Nat. Rev. Neurosci. 12:752. $10.1038 / \mathrm{nrn} 3122$.

[22] Botella C., Bretón-López J., Quero S., Baños R. M., García-Palacios A. (2010). Treating cockroach phobia with augmented reality. Behav. Ther. 41 401-413. 10.1016/j.beth.2009.07.002.

[23] Subhransu M. A. (2006). Comparison of Feature Descriptors, University of California at Berkeley, Department of EECS, University of California, Berkeley

[24] Rother, C., Kolmogorov, V. (2012). GrabCut-Interactive Foreground Extraction using Iterated Graph Cut. Microsoft Research Cambridge, UK, Andrew Blake.

[25] Saphiro L., Stockman G. (2001). Computer Vision, pp. 279-325, New Jersey, Prentice-Hall, ISBN 0-13-030796-3.

[26] Lorensen, William E.; Cline, Harvey E. (1987). Marching cubes: A high resolution 3D surface construction algorithm. ACM SIGGRAPH Computer Graphics. 21 (4): 163169. 10.1145/37402.37422.

[27] Unity. (2019). La plataforma de AR líder en el mundo. USA: Unity Technologies. Recuperado de https://unity3d.com/es/partners/Vuforia.

[28] Vuforia. (2018). Attach digital content to specific objects. USA: PTC Inc., Recuperado de https://engine.vuforia.com/features.

[29] Unity Technologies. (2019). Unity User Manual (2019.2): Types of light, USA: Unity Technologies. Recuperado de https://docs.unity3d.com/Manual/Lighting.html.
[30] Unity Technologies. (2019). Unity User Manual (2019.2): Line Renderer, USA: Unity Technologies. Recuperado de https://docs.unity3d.com/Manual/class-

LineRenderer.html.

[31] Vuforia. (2018). Privacy Policy \& EndUser License Agreement (EULA). USA: PTC Inc., Recuperado de https://www.goodnightlad.com/privacypolicy.html.

[32] Van de Kerckhove, E. (2019). HTC Vive Tutorial for Unity. USA: Razeware LLC, Recuperado de https://www.raywenderlich.com/9189-htc-vivetutorial-for-unity.

[33] Vergara D, Rubio MP, Lorenzo M. (2017). On the Design of Virtual Reality Learning Environments in Engineering. Multimodal Technologies and Interaction.; 1(2):11, 10.3390/mti1020011. 